\title{
A New Iterative Algorithm for the Set of Fixed-Point Problems of Nonexpansive Mappings and the Set of Equilibrium Problem and Variational Inequality Problem
}

\begin{abstract}
Atid Kangtunyakarn
Department of Mathematics, Faculty of Science, King Mongkut's Institute of Technology Ladkrabang, Bangkok 10520, Thailand

Correspondence should be addressed to Atid Kangtunyakarn, beawrock@hotmail.com

Received 7 October 2010; Revised 25 January 2011; Accepted 11 February 2011

Academic Editor: Norimichi Hirano

Copyright (c) 2011 Atid Kangtunyakarn. This is an open access article distributed under the Creative Commons Attribution License, which permits unrestricted use, distribution, and reproduction in any medium, provided the original work is properly cited.

We introduce a new iterative scheme and a new mapping generated by infinite family of nonexpansive mappings and infinite real number. By using both of these ideas, we obtain strong convergence theorem for finding a common element of the set of solution of equilibrium problem and the set of variational inequality and the set of fixed-point problems of infinite family of nonexpansive mappings. Moreover, we apply our main result to obtain strong convergence theorems for finding a common element of the set of solution of equilibrium problem and the set of variational inequality and the set of common fixed point of pseudocontractive mappings.
\end{abstract}

\section{Introduction}

Let $H$ be a real Hilbert space and let $C$ be a nonempty closed convex subset of $H$. Let $A$ : $C \rightarrow H$ be a nonlinear mapping and let $F: C \times C \rightarrow \mathbb{R}$ be a bifunction. A mapping $T$ of $H$ into itself is called nonexpansive if $\|T x-T y\| \leq\|x-y\|$ for all $x, y \in H$. We denote by $F(T)$ the set of fixed points of $T$ (i.e., $F(T)=\{x \in H: T x=x\}$ ). Goebel and Kirk [1] showed that $F(T)$ is always closed convex, and also nonempty provided $T$ has a bounded trajectory.

A bounded linear operator $A$ on $H$ is called strongly positive with coefficient $\bar{\gamma}$ if there is a constant $\bar{\gamma}>0$ with the property

$$
\langle A x, x\rangle \geq \bar{\gamma}\|x\|^{2}
$$


The equilibrium problem for $F$ is to find $x \in C$, such that

$$
F(x, y) \geq 0, \quad \forall y \in C
$$

The set of solutions of (1.2) is denoted by $\mathrm{EP}(F)$. Many problems in physics, optimization, and economics are seeking some elements of $\operatorname{EP}(F)$, see $[2,3]$. Several iterative methods have been proposed to solve the equilibrium problem, see, for instance, [2-4]. In 2005, Combettes and Hirstoaga [3] introduced an iterative scheme for finding the best approximation to the initial data when $\mathrm{EP}(F)$ is nonempty and proved a strong convergence theorem.

The variational inequality problem is to find a point $u \in C$, such that

$$
\langle v-u, A u\rangle \geq 0, \quad \forall v \in C
$$

The set of solutions of the variational inequality is denoted by $\operatorname{VI}(C, A)$. Numerous problems in physics, optimization, variational inequalities, minimax problems, the Nash equilibrium problem in noncooperative games reduce to find element of (1.2) and (1.3).

A mapping $A$ of $C$ into $H$ is called inverse-strongly monotone, see [5], if there exists a positive real number $\alpha$, such that

$$
\langle x-y, A x-A y\rangle \geq \alpha\|A x-A y\|^{2}
$$

for all $x, y \in C$.

The problem of finding a common fixed point of a family of nonexpansive mappings has been studied by many authors. The well-known convex feasibility problem reduces to finding a point in the intersection of the fixed-point sets of a family of nonexpansive mapping (see $[6,7])$.

The problem of finding a common element of $\mathrm{EP}(F)$ and the set of all common fixed points of a family of nonexpansive mappings is of wide interdisciplinary interest and importance. Many iterative methods are purposed for finding a common element of the solutions of the equilibrium problem and fixed-point problem of nonexpansive mappings, see [8-10].

In 2007, S. Takahashi and W. Takahashi [10] introduced a general iterative method for finding a common element of $\operatorname{EP}(F, A)$ and $F(T)$. They defined $\left\{x_{n}\right\}$ in the following way:

$$
\begin{gathered}
u, x_{1} \in C, \text { arbitrarily; } \\
F\left(z_{n}, y\right)+\frac{1}{\lambda_{n}}\left\langle y-z_{n}, z_{n}-x_{n}\right\rangle \geq 0, \quad \forall y \in C, \\
x_{n+1}=\beta_{n} f\left(x_{n}\right)+\left(1-\beta_{n}\right) S z_{n}, \quad \forall n \in \mathbb{N},
\end{gathered}
$$

where $\left\{\beta_{n}\right\} \subset[0,1]$, and proved strong convergence of the scheme (1.5) to $z \in F(T) \bigcap \operatorname{EP}(F)$, where $z=P_{F(S) \cap \operatorname{EP}(F)} f(z)$ in the framework of a Hilbert space, under some suitable conditions on $\left\{\beta_{n}\right\},\left\{\lambda_{n}\right\}$ and bifunction $F$.

In this paper, by motive of (1.5), we prove strong convergence theorem for finding a common element of the set of solution of equilibrium problem and the set of variational 
inequality and the set of fixed-point problems by using a new mapping generated by infinite family of nonexpansive mapping and infinite real number. Moreover, we apply our main result to obtain strong convergence theorems for finding a common element of the set of solution of equilibrium problem and the set of variational inequality and the set of common fixed point of pseudocontractive mappings.

\section{Preliminaries}

In this section, we collect and give some useful lemmas that will be used for our main result in the next section.

Let $C$ be closed convex subset of a real Hilbert space $H$, and let $P_{C}$ be the metric projection of $H$ onto $C$, that is, for $x \in H, P_{C} x$ satisfies the property

$$
\left\|x-P_{C} x\right\|=\min _{y \in C}\|x-y\|
$$

The following characterizes the projection $P_{C}$.

Lemma 2.1 (see [11]). Given $x \in H$ and $y \in C$, then $P_{C} x=y$ if and only if there holds the inequality

$$
\langle x-y, y-z\rangle \geq 0, \quad \forall z \in C
$$

Lemma 2.2 (see [12]). Let $E$ be a uniformly convex Banach space, let $C$ be a nonempty closed convex subset of $E$, and let $S: C \rightarrow C$ be a nonexpansive mapping, then $I-S$ is demiclosed at zero.

Lemma 2.3 (see [13]). Let $\left\{s_{n}\right\}$ be a sequence of nonnegative real numbers satisfying

$$
s_{n+1}=\left(1-\alpha_{n}\right) s_{n}+\delta_{n}, \quad \forall n \geq 0,
$$

where $\left\{\alpha_{n}\right\}$ is a sequence in $(0,1)$ and $\left\{\delta_{n}\right\}$ is a sequence, such that

(1) $\sum_{n=1}^{\infty} \alpha_{n}=\infty$,

(2) $\lim \sup _{n \rightarrow \infty} \delta_{n} / \alpha_{n} \leq 0$ or $\sum_{n=1}^{\infty}\left|\delta_{n}\right|<\infty$

then $\lim _{n \rightarrow \infty} s_{n}=0$.

For solving the equilibrium problem for a bifunction $F: C \times C \rightarrow \mathbb{R}$, let us assume that $F$ satisfies the following conditions:

(A1) $F(x, x)=0$, for all $x \in C$,

(A2) $F$ is monotone, that is, $F(x, y)+F(y, x) \leq 0$, for all $x, y \in C$, 
(A3) for all $x, y, z \in C$,

$$
\lim _{t \rightarrow 0^{+}} F(t z+(1-t) x, y) \leq F(x, y)
$$

(A4) for all $x \in C, y \mapsto F(x, y)$ is convex and lower semicontinuous.

The following lemma appears implicitly in [2].

Lemma 2.4 (see [2]). Let $C$ be a nonempty closed convex subset of $H$, and let $F$ be a bifunction of $C \times C$ into $\mathbb{R}$ satisfying (A1)-(A4). Let $r>0$ and $x \in H$, then there exists $z \in C$, such that

$$
F(z, y)+\frac{1}{r}\langle y-z, z-x\rangle
$$

for all $x \in C$.

Lemma 2.5 (see [3]). Assume that $F: C \times C \rightarrow \mathbb{R}$ satisfies (A1)-(A4). For $r>0$ and $x \in H$, define a mapping $T_{r}: H \rightarrow C$ as follows:

$$
T_{r}(x)=\left\{z \in C: F(z, y)+\frac{1}{r}\langle y-z, z-x\rangle \geq 0, \forall y \in C\right\}
$$

for all $z \in H$, then the following hold:

(1) $T_{r}$ is single valued,

(2) $T_{r}$ is firmly nonexpansive, that is,

$$
\left\|T_{r}(x)-T_{r}(y)\right\|^{2} \leq\left\langle T_{r}(x)-T_{r}(y), x-y\right\rangle \quad \forall x, y \in H
$$

(3) $F\left(T_{r}\right)=\mathrm{EP}(F)$,

(4) $\mathrm{EP}(F)$ is closed and convex.

Lemma 2.6 (see [14]). Let $H$ be a Hibert space, let $C$ be a nonempty closed convex subset of $H$, and let $A$ be a mapping of $C$ into $H$. Let $u \in C$, then for $\lambda>0$,

$$
u=P_{C}(I-\lambda A) u \Longleftrightarrow u \in \operatorname{VI}(C, A),
$$

where $P_{C}$ is the metric projection of $H$ onto $C$. 
Definition 2.7. Let $C$ be a nonempty convex subset of a real Hilbert space. Let $T_{i}, i=1,2, \ldots$ be mappings of $C$ into itself. For each $j=1,2, \ldots$, let $\alpha_{j}=\left(\alpha_{1}^{j}, \alpha_{2}^{j}, \alpha_{3}^{j}\right) \in I \times I \times I$ where $I \in[0,1]$ and $\alpha_{1}^{j}+\alpha_{2}^{j}+\alpha_{3}^{j}=1$. For every $n \in \mathbb{N}$, we define the mapping $S_{n}: C \rightarrow C$ as follows:

$$
\begin{aligned}
U_{n, n+1} & =I, \\
U_{n, n} & =\alpha_{1}^{n} T_{n} U_{n, n+1}+\alpha_{2}^{n} U_{n, n+1}+\alpha_{3}^{n} I, \\
U_{n, n-1}= & \alpha_{1}^{n-1} T_{n-1} U_{n, n}+\alpha_{2}^{n-1} U_{n, n}+\alpha_{3}^{n-1} I, \\
& \vdots \\
U_{n, k+1} & =\alpha_{1}^{k+1} T_{k+1} U_{n, k+2}+\alpha_{2}^{k+1} U_{n, k+2}+\alpha_{3}^{k+1} I, \\
U_{n, k}= & \alpha_{1}^{k} T_{k} U_{n, k+1}+\alpha_{2}^{k} U_{n, k+1}+\alpha_{3}^{k} I, \\
& \vdots \\
U_{n, 2}= & \alpha_{1}^{2} T_{2} U_{n, 3}+\alpha_{2}^{2} U_{n, 3}+\alpha_{3}^{2} I, \\
S_{n}= & U_{n, 1}=\alpha_{1}^{1} T_{1} U_{n, 2}+\alpha_{2}^{1} U_{n, 2}+\alpha_{3}^{1} I .
\end{aligned}
$$

This mapping is called S-mapping generated by $T_{n}, \ldots, T_{1}$ and $\alpha_{n}, \alpha_{n-1}, \ldots, \alpha_{1}$.

Lemma 2.8. Let $C$ be a nonempty closed convex subset of a real Hilbert space. Let $\left\{T_{i}\right\}_{i=1}^{\infty}$ be nonexpansive mappings of $C$ into itself with $\bigcap_{i=1}^{\infty} F\left(T_{i}\right) \neq \emptyset$, and let $\alpha_{j}=\left(\alpha_{1}^{j}, \alpha_{2}^{j}, \alpha_{3}^{j}\right) \in I \times I \times I$, where $I=[0,1], \alpha_{1}^{j}+\alpha_{2}^{j}+\alpha_{3}^{j}=1, \alpha_{1}^{j}+\alpha_{2}^{j} \leq b<1$, and $\alpha_{1}^{j}, \alpha_{2}^{j}, \alpha_{3}^{j} \in(0,1)$, for all $j=1,2, \ldots$ For every $n \in \mathbb{N}$, let $S_{n}$ be $S$-mapping generated by $T_{n}, \ldots, T_{1}$ and $\alpha_{n}, \alpha_{n-1}, \ldots, \alpha_{1}$, then for every $x \in C$ and $k \in \mathbb{N}, \lim _{n \rightarrow \infty} U_{n, k} x$ exists.

Proof. Let $x \in C$ and $y \in \bigcap_{i=1}^{\infty} F\left(T_{i}\right)$. Fix $k \in \mathbb{N}$, then for every $n \in \mathbb{N}$ with $n \geq k$, we have

$$
\begin{aligned}
\left\|U_{n+1, k} x-U_{n, k} x\right\|^{2}= & \| \alpha_{1}^{k} T_{k} U_{n+1, k+1} x+\alpha_{2}^{k} U_{n+1, k+1} x+\alpha_{3}^{k} x \\
& -\alpha_{1}^{k} T_{k} U_{n, k+1} x-\alpha_{2}^{k} U_{n, k+1} x-\alpha_{3}^{k} x \|^{2} \\
= & \left\|\alpha_{1}^{k}\left(T_{k} U_{n+1, k+1} x-T_{k} U_{n, k+1} x\right)+\alpha_{2}^{k}\left(U_{n+1, k+1} x-U_{n, k+1} x\right)\right\|^{2} \\
\leq & \alpha_{1}^{k}\left\|T_{k} U_{n+1, k+1} x-T_{k} U_{n, k+1} x\right\|^{2}+\alpha_{2}^{k}\left\|U_{n+1, k+1} x-U_{n, k+1} x\right\|^{2} \\
\leq & \alpha_{1}^{k}\left\|U_{n+1, k+1} x-U_{n, k+1} x\right\|^{2}+\alpha_{2}^{k}\left\|U_{n+1, k+1} x-U_{n, k+1} x\right\|^{2} \\
\leq & \left(1-\alpha_{3}^{k}\right)\left\|U_{n+1, k+1} x-U_{n, k+1} x\right\|^{2}
\end{aligned}
$$




$$
\begin{aligned}
& \leq \prod_{j=k}^{n}\left(1-\alpha_{3}^{j}\right)\left\|U_{n+1, n+1} x-U_{n, n+1} x\right\|^{2} \\
& =\prod_{j=k}^{n}\left(1-\alpha_{3}^{j}\right)\left\|\alpha_{1}^{n+1} T_{n+1} U_{n+1, n+2} x+\alpha_{2}^{n+1} U_{n+1, n+2} x+\alpha_{3}^{n+1} x-x\right\|^{2} \\
& =\prod_{j=k}^{n}\left(1-\alpha_{3}^{j}\right)\left\|\alpha_{1}^{n+1} T_{n+1} x+\left(1-\alpha_{1}^{n+1}\right) x-x\right\|^{2} \\
& =\prod_{j=k}^{n}\left(1-\alpha_{3}^{j}\right)\left\|\alpha_{1}^{n+1}\left(T_{n+1} x-x\right)\right\|^{2} \\
& \leq \prod_{j=k}^{n}\left(1-\alpha_{3}^{j}\right)\left(\left\|T_{n+1} x-y\right\|+\|y-x\|\right)^{2} \\
& \leq \prod_{j=k}^{n}\left(1-\alpha_{3}^{j}\right)(\|x-y\|+\|y-x\|)^{2} \\
& \leq \prod_{j=k}^{n}\left(1-\alpha_{3}^{j}\right)(2\|x-y\|)^{2} \\
& \leq b^{n-(k-1)}(2\|x-y\|)^{2} .
\end{aligned}
$$

It follows that

$$
\begin{aligned}
\left\|U_{n+1, k} x-U_{n, k} x\right\| & \leq b^{(n-(k-1)) / 2}(2\|x-y\|) \\
& =\frac{b^{n / 2}}{b^{(k-1) / 2}}(2\|x-y\|) \\
& =\frac{a^{n}}{a^{k-1}} M,
\end{aligned}
$$

where $a=b^{1 / 2} \in(0,1)$ and $M=2\|x-y\|$.

For any $k, n, p \in \mathbb{N}, p>0, n \geq k$, we have

$$
\begin{aligned}
\left\|U_{n+p, k} x-U_{n, k} x\right\| \leq & \left\|U_{n+p, k} x-U_{n+p-1, k} x\right\|+\left\|U_{n+p-1, k} x-U_{n+p-2, k} x\right\| \\
& +\cdots+\left\|U_{n+1, k} x-U_{n, k} x\right\| \\
= & \sum_{j=n}^{n+p-1}\left\|U_{j+1, k} x-U_{j, k} x\right\| \\
\leq & \sum_{j=n}^{n+p-1} \frac{a^{j}}{a^{k-1}} M \\
\leq & \frac{a^{n}}{(1-a) a^{k-1}} M .
\end{aligned}
$$


Since $a \in(0,1)$, we have $\lim _{n \rightarrow \infty} a^{n}=0$. From (2.12), we have that $\left\{U_{n, k} x\right\}$ is a Cauchy sequence. Hence, $\lim _{n \rightarrow \infty} U_{n, k} x$ exists.

For every $k \in \mathbb{N}$ and $x \in C$, we define mapping $U_{\infty, k}$ and $S: C \rightarrow C$ as follows:

$$
\begin{gathered}
\lim _{n \rightarrow \infty} U_{n, k} x=U_{\infty, k} x, \\
\lim _{n \rightarrow \infty} S_{n} x=\lim _{n \rightarrow \infty} U_{n, 1} x=S x .
\end{gathered}
$$

Such a mapping $S$ is called $S$-mapping generated by $T_{n}, T_{n-1}, \ldots$ and $\alpha_{n}, \alpha_{n-1}, \ldots$

Remark 2.9. For each $n \in \mathbb{N}, S_{n}$ is nonexpansive and $\lim _{n \rightarrow \infty} \sup _{x \in D}\left\|S_{n} x-S x\right\|=0$ for every bounded subset $D$ of $C$. To show this, let $x, y \in C$ and $D$ be a bounded subset of $C$, then we have

$$
\begin{aligned}
\left\|S_{n} x-S_{n} y\right\|^{2} & =\left\|\alpha_{1}^{1}\left(T_{1} U_{n, 2} x-T_{1} U_{n, 2} y\right)+\alpha_{2}^{1}\left(U_{n, 2} x-U_{n, 2} y\right)+\alpha_{3}^{1}(x-y)\right\|^{2} \\
& \leq \alpha_{1}^{1}\left\|T_{1} U_{n, 2} x-T_{1} U_{n, 2} y\right\|^{2}+\alpha_{2}^{1}\left\|U_{n, 2} x-U_{n, 2} y\right\|^{2}+\alpha_{3}^{1}\|x-y\|^{2} \\
& \leq \alpha_{1}^{1}\left\|U_{n, 2} x-U_{n, 2} y\right\|^{2}+\alpha_{2}^{1}\left\|U_{n, 2} x-U_{n, 2} y\right\|^{2}+\alpha_{3}^{1}\|x-y\|^{2} \\
& =\left(1-\alpha_{3}^{1}\right)\left\|U_{n, 2} x-U_{n, 2} y\right\|^{2}+\alpha_{3}^{1}\|x-y\|^{2} \\
& \leq\left(1-\alpha_{3}^{1}\right)\left(\left(1-\alpha_{3}^{2}\right)\left\|U_{n, 3} x-U_{n, 3} y\right\|^{2}+\alpha_{3}^{2}\|x-y\|^{2}\right)+\alpha_{3}^{1}\|x-y\|^{2} \\
& =\left(1-\alpha_{3}^{1}\right)\left(1-\alpha_{3}^{2}\right)\left\|U_{n, 3} x-U_{n, 3} y\right\|^{2}+\alpha_{3}^{2}\left(1-\alpha_{3}^{1}\right)\|x-y\|^{2}+\alpha_{3}^{1}\|x-y\|^{2} \\
& =\prod_{j=1}^{2}\left(1-\alpha_{3}^{j}\right)\left\|U_{n, 3} x-U_{n, 3} y\right\|^{2}+\left(1-\prod_{j=1}^{2}\left(1-\alpha_{3}^{j}\right)\right)\|x-y\|^{2} \\
& \vdots \\
& \leq \prod_{j=1}^{2}\left(1-\alpha_{3}^{j}\right)\left\|U_{n, n+1} x-U_{n, n+1} y\right\|^{2}+\left(1-\prod_{j=1}^{2}\left(1-\alpha_{3}^{j}\right)\right)\|x-y\|^{2} \\
& \|x-y\|^{2} .
\end{aligned}
$$


Then, we have that $S: C \rightarrow C$ is also nonexpansive, Indeed, observe that for each $x, y \in C$,

$$
\|S x-S y\|=\lim _{n \rightarrow \infty}\left\|S_{n} x-S_{n} y\right\| \leq\|x-y\| .
$$

By (2.11), we have

$$
\begin{aligned}
\left\|S_{n+1} x-S_{n} x\right\| & =\left\|U_{n+1,1} x-U_{n, 1} x\right\| \\
& \leq a^{n} M .
\end{aligned}
$$

This implies that for $m>n$ and $x \in D$,

$$
\begin{aligned}
\left\|S_{m} x-S_{n} x\right\| & \leq \sum_{j=n}^{m-1}\left\|S_{j+1} x-S_{j} x\right\| \\
& \leq \sum_{j=n}^{m-1} a^{j} M \\
& \leq \frac{a^{n}}{1-a} M .
\end{aligned}
$$

By letting $m \rightarrow \infty$, for any $x \in D$, we have

$$
\left\|S x-S_{n} x\right\| \leq \frac{a^{n}}{1-a} M
$$

It follows that

$$
\lim _{n \rightarrow \infty} \sup _{x \in D}\left\|S_{n} x-S x\right\|=0 .
$$

Lemma 2.10. Let $C$ be a nonempty closed convex subset of a real Hilbert space. Let $\left\{T_{i}\right\}_{i=1}^{\infty}$ be nonexpansive mappings of $C$ into itself with $\bigcap_{i=1}^{\infty} F\left(T_{i}\right) \neq \emptyset$, and let $\alpha_{j}=\left(\alpha_{1}^{j}, \alpha_{2}^{j}, \alpha_{3}^{j}\right) \in I \times I \times I$, where $I=[0,1], \alpha_{1}^{j}+\alpha_{2}^{j}+\alpha_{3}^{j}=1, \alpha_{1}^{j}+\alpha_{2}^{j} \leq b<1$, and $\alpha_{1}^{j}, \alpha_{2}^{j}, \alpha_{3}^{j} \in(0,1)$ for all $j=1,2, \ldots$. For every $n \in \mathbb{N}$, let $S_{n}$ and $S$ be $S$-mappings generated by $T_{n}, \ldots, T_{1}$ and $\alpha_{n}, \alpha_{n-1}, \ldots, \alpha_{1}$ and $T_{n}, T_{n-1}, \ldots$, and $\alpha_{n}, \alpha_{n-1}, \ldots$, respectively, then $F(S)=\bigcap_{i=1}^{\infty} F\left(T_{i}\right)$. 
Proof. It is easy to see that $\bigcap_{i=1}^{\infty} F\left(T_{i}\right) \subseteq F(S)$. For every $n, k \in \mathbb{N}$, with $n \geq k$, let $x_{0} \in F(S)$ and $x^{*} \in \bigcap_{i=1}^{\infty} F\left(T_{i}\right)$, then we have

$$
\begin{aligned}
& \left\|S_{n} x_{0}-x^{*}\right\|^{2}=\left\|\alpha_{1}^{1}\left(T_{1} U_{n, 2} x_{0}-x^{*}\right)+\alpha_{2}^{1}\left(U_{n, 2} x_{0}-x^{*}\right)+\alpha_{3}^{1}\left(x_{0}-x^{*}\right)\right\|^{2} \\
& \leq \alpha_{1}^{1}\left\|T_{1} U_{n, 2} x_{0}-x^{*}\right\|^{2}+\alpha_{2}^{1}\left\|U_{n, 2} x_{0}-x^{*}\right\|^{2}+\alpha_{3}^{1}\left\|x_{0}-x^{*}\right\|^{2} \\
& -\alpha_{1}^{1} \alpha_{2}^{1}\left\|T_{1} U_{n, 2} x_{0}-U_{n, 2} x_{0}\right\|^{2}-\alpha_{2}^{1} \alpha_{3}^{1}\left\|U_{n, 2} x_{0}-x_{0}\right\|^{2} \\
& \leq \alpha_{1}^{1}\left\|U_{n, 2} x_{0}-x^{*}\right\|^{2}+\alpha_{2}^{1}\left\|U_{n, 2} x_{0}-x^{*}\right\|^{2} \\
& +\alpha_{3}^{1}\left\|x_{0}-x^{*}\right\|^{2}-\alpha_{1}^{1} \alpha_{2}^{1}\left\|T_{1} U_{n, 2} x_{0}-U_{n, 2} x_{0}\right\|^{2}-\alpha_{2}^{1} \alpha_{3}^{1}\left\|U_{n, 2} x_{0}-x_{0}\right\|^{2} \\
& =\left(1-\alpha_{3}^{1}\right)\left\|U_{n, 2} x_{0}-x^{*}\right\|^{2}+\alpha_{3}^{1}\left\|x_{0}-x^{*}\right\|^{2} \\
& -\alpha_{1}^{1} \alpha_{2}^{1}\left\|T_{1} U_{n, 2} x_{0}-U_{n, 2} x_{0}\right\|^{2}-\alpha_{2}^{1} \alpha_{3}^{1}\left\|U_{n, 2} x_{0}-x_{0}\right\|^{2} \\
& \leq\left(1-\alpha_{3}^{1}\right)\left(\left(1-\alpha_{3}^{2}\right)\left\|U_{n, 3} x_{0}-x^{*}\right\|^{2}+\alpha_{3}^{2}\left\|x_{0}-x^{*}\right\|^{2}\right. \\
& \left.-\alpha_{1}^{2} \alpha_{2}^{2}\left\|T_{2} U_{n, 3} x_{0}-U_{n, 3} x_{0}\right\|^{2}-\alpha_{2}^{2} \alpha_{3}^{2}\left\|U_{n, 3} x_{0}-x_{0}\right\|^{2}\right)+\alpha_{3}^{1}\left\|x_{0}-x^{*}\right\|^{2} \\
& -\alpha_{1}^{1} \alpha_{2}^{1}\left\|T_{1} U_{n, 2} x_{0}-U_{n, 2} x_{0}\right\|^{2}-\alpha_{2}^{1} \alpha_{3}^{1}\left\|U_{n, 2} x_{0}-x_{0}\right\|^{2} \\
& =\left(1-\alpha_{3}^{1}\right)\left(1-\alpha_{3}^{2}\right)\left\|U_{n, 3} x_{0}-x^{*}\right\|^{2}+\alpha_{3}^{2}\left(1-\alpha_{3}^{1}\right)\left\|x_{0}-x^{*}\right\|^{2}+\alpha_{3}^{1}\left\|x_{0}-x^{*}\right\|^{2} \\
& -\alpha_{1}^{2} \alpha_{2}^{2}\left(1-\alpha_{3}^{1}\right)\left\|T_{2} U_{n, 3} x_{0}-U_{n, 3} x_{0}\right\|^{2}-\alpha_{2}^{2} \alpha_{3}^{2}\left(1-\alpha_{3}^{1}\right)\left\|U_{n, 3} x_{0}-x_{0}\right\|^{2} \\
& -\alpha_{1}^{1} \alpha_{2}^{1}\left\|T_{1} U_{n, 2} x_{0}-U_{n, 2} x_{0}\right\|^{2}-\alpha_{2}^{1} \alpha_{3}^{1}\left\|U_{n, 2} x_{0}-x_{0}\right\|^{2} \\
& =\prod_{j=1}^{2}\left(1-\alpha_{3}^{j}\right)\left\|U_{n, 3} x_{0}-x^{*}\right\|^{2}+\left(1-\prod_{j=1}^{2}\left(1-\alpha_{3}^{j}\right)\right)\left\|x_{0}-x^{*}\right\|^{2} \\
& -\alpha_{1}^{2} \alpha_{2}^{2}\left(1-\alpha_{3}^{1}\right)\left\|T_{2} U_{n, 3} x_{0}-U_{n, 3} x_{0}\right\|^{2}-\alpha_{2}^{2} \alpha_{3}^{2}\left(1-\alpha_{3}^{1}\right)\left\|U_{n, 3} x_{0}-x_{0}\right\|^{2} \\
& -\alpha_{1}^{1} \alpha_{2}^{1}\left\|T_{1} U_{n, 2} x_{0}-U_{n, 2} x_{0}\right\|^{2}-\alpha_{2}^{1} \alpha_{3}^{1}\left\|U_{n, 2} x_{0}-x_{0}\right\|^{2} \\
& \leq \prod_{j=1}^{2}\left(1-\alpha_{3}^{j}\right)\left(\left(1-\alpha_{3}^{3}\right)\left\|U_{n, 4} x_{0}-x^{*}\right\|^{2}+\alpha_{3}^{3}\left\|x_{0}-x^{*}\right\|^{2}\right. \\
& \left.-\alpha_{1}^{3} \alpha_{2}^{3}\left\|T_{3} U_{n, 4} x_{0}-U_{n, 4} x_{0}\right\|^{2}-\alpha_{2}^{3} \alpha_{3}^{3}\left\|U_{n, 4} x_{0}-x_{0}\right\|^{2}\right) \\
& +\left(1-\prod_{j=1}^{2}\left(1-\alpha_{3}^{j}\right)\right)\left\|x_{0}-x^{*}\right\|^{2}-\alpha_{1}^{2} \alpha_{2}^{2}\left(1-\alpha_{3}^{1}\right)\left\|T_{2} U_{n, 3} x_{0}-U_{n, 3} x_{0}\right\|^{2} \\
& -\alpha_{2}^{2} \alpha_{3}^{2}\left(1-\alpha_{3}^{1}\right)\left\|U_{n, 3} x_{0}-x_{0}\right\|^{2}-\alpha_{1}^{1} \alpha_{2}^{1}\left\|T_{1} U_{n, 2} x_{0}-U_{n, 2} x_{0}\right\|^{2}-\alpha_{2}^{1} \alpha_{3}^{1}\left\|U_{n, 2} x_{0}-x_{0}\right\|^{2} \\
& =\prod_{j=1}^{2}\left(1-\alpha_{3}^{j}\right)\left(1-\alpha_{3}^{3}\right)\left\|U_{n, 4} x_{0}-x^{*}\right\|^{2}+\alpha_{3}^{3} \prod_{j=1}^{2}\left(1-\alpha_{3}^{j}\right)\left\|x_{0}-x^{*}\right\|^{2}
\end{aligned}
$$




$$
\begin{aligned}
& -\alpha_{1}^{3} \alpha_{2}^{3} \prod_{j=1}^{2}\left(1-\alpha_{3}^{j}\right)\left\|T_{3} U_{n, 4} x_{0}-U_{n, 4} x_{0}\right\|^{2} \\
& -\alpha_{2}^{3} \alpha_{3}^{3} \prod_{j=1}^{2}\left(1-\alpha_{3}^{j}\right)\left\|U_{n, 4} x_{0}-x_{0}\right\|^{2}+\left(1-\prod_{j=1}^{2}\left(1-\alpha_{3}^{j}\right)\right)\left\|x_{0}-x^{*}\right\|^{2} \\
& -\alpha_{1}^{2} \alpha_{2}^{2}\left(1-\alpha_{3}^{1}\right)\left\|T_{2} U_{n, 3} x_{0}-U_{n, 3} x_{0}\right\|^{2}-\alpha_{2}^{2} \alpha_{3}^{2}\left(1-\alpha_{3}^{1}\right)\left\|U_{n, 3} x_{0}-x_{0}\right\|^{2} \\
& -\alpha_{1}^{1} \alpha_{2}^{1}\left\|T_{1} U_{n, 2} x_{0}-U_{n, 2} x_{0}\right\|^{2}-\alpha_{2}^{1} \alpha_{3}^{1}\left\|U_{n, 2} x_{0}-x_{0}\right\|^{2} \\
& =\prod_{j=1}^{3}\left(1-\alpha_{3}^{j}\right)\left\|U_{n, 4} x_{0}-x^{*}\right\|^{2}+\left(1-\prod_{j=1}^{3}\left(1-\alpha_{3}^{j}\right)\right)\left\|x_{0}-x^{*}\right\|^{2} \\
& -\alpha_{1}^{3} \alpha_{2}^{3} \prod_{j=1}^{2}\left(1-\alpha_{3}^{j}\right)\left\|T_{3} U_{n, 4} x_{0}-U_{n, 4} x_{0}\right\|^{2}-\alpha_{2}^{3} \alpha_{3}^{3} \prod_{j=1}^{2}\left(1-\alpha_{3}^{j}\right)\left\|U_{n, 4} x_{0}-x_{0}\right\|^{2} \\
& -\alpha_{1}^{2} \alpha_{2}^{2}\left(1-\alpha_{3}^{1}\right)\left\|T_{2} U_{n, 3} x_{0}-U_{n, 3} x_{0}\right\|^{2}-\alpha_{2}^{2} \alpha_{3}^{2}\left(1-\alpha_{3}^{1}\right)\left\|U_{n, 3} x_{0}-x_{0}\right\|^{2} \\
& -\alpha_{1}^{1} \alpha_{2}^{1}\left\|T_{1} U_{n, 2} x_{0}-U_{n, 2} x_{0}\right\|^{2}-\alpha_{2}^{1} \alpha_{3}^{1}\left\|U_{n, 2} x_{0}-x_{0}\right\|^{2} \\
& \leq \prod_{j=1}^{k+1}\left(1-\alpha_{3}^{j}\right)\left\|U_{n, k+2} x_{0}-x^{*}\right\|^{2}+\left(1-\prod_{j=1}^{k+1}\left(1-\alpha_{3}^{j}\right)\right)\left\|x_{0}-x^{*}\right\|^{2} \\
& -\alpha_{1}^{k+1} \alpha_{2}^{k+1} \prod_{j=1}^{k}\left(1-\alpha_{3}^{j}\right)\left\|T_{k+1} U_{n, k+2} x_{0}-U_{n, k+2} x_{0}\right\|^{2} \\
& -\alpha_{2}^{k+1} \alpha_{3}^{k+1} \prod_{j=1}^{k}\left(1-\alpha_{3}^{j}\right)\left\|U_{n, k+2} x_{0}-x_{0}\right\|^{2} \\
& -\alpha_{1}^{k} \alpha_{2}^{k} \prod_{j=1}^{k-1}\left(1-\alpha_{3}^{j}\right)\left\|T_{k} U_{n, k+1} x_{0}-U_{n, k+1} x_{0}\right\|^{2} \\
& -\alpha_{2}^{k} \alpha_{3}^{k} \prod_{j=1}^{k-1}\left(1-\alpha_{3}^{j}\right)\left\|U_{n, k+1} x_{0}-x_{0}\right\|^{2} \\
& -\alpha_{1}^{k-1} \alpha_{2}^{k-1} \prod_{j=1}^{k-2}\left(1-\alpha_{3}^{j}\right)\left\|T_{k-1} U_{n, k} x_{0}-U_{n, k} x_{0}\right\|^{2} \\
& -\alpha_{2}^{k-1} \alpha_{3}^{k-1} \prod_{j=1}^{k-2}\left(1-\alpha_{3}^{j}\right)\left\|U_{n, k} x_{0}-x_{0}\right\|^{2}
\end{aligned}
$$


Abstract and Applied Analysis

$$
\begin{aligned}
& -\alpha_{1}^{3} \alpha_{2}^{3} \prod_{j=1}^{2}\left(1-\alpha_{3}^{j}\right)\left\|T_{3} U_{n, 4} x_{0}-U_{n, 4} x_{0}\right\|^{2}-\alpha_{2}^{3} \alpha_{3}^{3} \prod_{j=1}^{2}\left(1-\alpha_{3}^{j}\right)\left\|U_{n, 4} x_{0}-x_{0}\right\|^{2} \\
& -\alpha_{1}^{2} \alpha_{2}^{2}\left(1-\alpha_{3}^{1}\right)\left\|T_{2} U_{n, 3} x_{0}-U_{n, 3} x_{0}\right\|^{2}-\alpha_{2}^{2} \alpha_{3}^{2}\left(1-\alpha_{3}^{1}\right)\left\|U_{n, 3} x_{0}-x_{0}\right\|^{2} \\
& -\alpha_{1}^{1} \alpha_{2}^{1}\left\|T_{1} U_{n, 2} x_{0}-U_{n, 2} x_{0}\right\|^{2}-\alpha_{2}^{1} \alpha_{3}^{1}\left\|U_{n, 2} x_{0}-x_{0}\right\|^{2} \\
& \vdots \\
& \leq \prod_{j=1}^{n}\left(1-\alpha_{3}^{j}\right)\left\|U_{n, n+1} x_{0}-x^{*}\right\|^{2}+\left(1-\prod_{j=1}^{n}\left(1-\alpha_{3}^{j}\right)\right)\left\|x_{0}-x^{*}\right\|^{2} \\
& -\alpha_{1}^{n} \alpha_{2}^{n} \prod_{j=1}^{n-1}\left(1-\alpha_{3}^{j}\right)\left\|T_{n} U_{n, n+1} x_{0}-U_{n, n+1} x_{0}\right\|^{2} \\
& -\alpha_{2}^{n} \alpha_{3}^{n} \prod_{j=1}^{n-1}\left(1-\alpha_{3}^{j}\right)\left\|U_{n, n+1} x_{0}-x_{0}\right\|^{2} \\
& \vdots \\
& -\alpha_{1}^{k+1} \alpha_{2}^{k+1} \prod_{j=1}^{k}\left(1-\alpha_{3}^{j}\right)\left\|T_{k+1} U_{n, k+2} x_{0}-U_{n, k+2} x_{0}\right\|^{2} \\
& -\alpha_{2}^{k+1} \alpha_{3}^{k+1} \prod_{j=1}^{k}\left(1-\alpha_{3}^{j}\right)\left\|U_{n, k+2} x_{0}-x_{0}\right\|^{2} \\
& -\alpha_{1}^{k} \alpha_{2}^{k} \prod_{j=1}^{k-1}\left(1-\alpha_{3}^{j}\right)\left\|T_{k} U_{n, k+1} x_{0}-U_{n, k+1} x_{0}\right\|^{2} \\
& -\alpha_{2}^{k} \alpha_{3}^{k} \prod_{j=1}^{k-1}\left(1-\alpha_{3}^{j}\right)\left\|U_{n, k+1} x_{0}-x_{0}\right\|^{2} \\
& -\alpha_{1}^{k-1} \alpha_{2}^{k-1} \prod_{j=1}^{k-2}\left(1-\alpha_{3}^{j}\right)\left\|T_{k-1} U_{n, k} x_{0}-U_{n, k} x_{0}\right\|^{2} \\
& -\alpha_{2}^{k-1} \alpha_{3}^{k-1} \prod_{j=1}^{k-2}\left(1-\alpha_{3}^{j}\right)\left\|U_{n, k} x_{0}-x_{0}\right\|^{2} \\
& \vdots \\
& -\alpha_{1}^{3} \alpha_{2}^{3} \prod_{j=1}^{2}\left(1-\alpha_{3}^{j}\right)\left\|T_{3} U_{n, 4} x_{0}-U_{n, 4} x_{0}\right\|^{2}-\alpha_{2}^{3} \alpha_{3}^{3} \prod_{j=1}^{2}\left(1-\alpha_{3}^{j}\right)\left\|U_{n, 4} x_{0}-x_{0}\right\|^{2} \\
& -\alpha_{1}^{2} \alpha_{2}^{2}\left(1-\alpha_{3}^{1}\right)\left\|T_{2} U_{n, 3} x_{0}-U_{n, 3} x_{0}\right\|^{2}-\alpha_{2}^{2} \alpha_{3}^{2}\left(1-\alpha_{3}^{1}\right)\left\|U_{n, 3} x_{0}-x_{0}\right\|^{2}
\end{aligned}
$$




$$
\begin{aligned}
& -\alpha_{1}^{1} \alpha_{2}^{1}\left\|T_{1} U_{n, 2} x_{0}-U_{n, 2} x_{0}\right\|^{2}-\alpha_{2}^{1} \alpha_{3}^{1}\left\|U_{n, 2} x_{0}-x_{0}\right\|^{2} \\
= & \left\|x_{0}-x^{*}\right\|^{2} \\
& -\alpha_{1}^{n} \alpha_{2}^{n} \prod_{j=1}^{n-1}\left(1-\alpha_{3}^{j}\right)\left\|T_{n} U_{n, n+1} x_{0}-U_{n, n+1} x_{0}\right\|^{2} \\
& \vdots \\
& -\alpha_{1}^{k+1} \alpha_{2}^{k+1} \prod_{j=1}^{k}\left(1-\alpha_{3}^{j}\right)\left\|T_{k+1} U_{n, k+2} x_{0}-U_{n, k+2} x_{0}\right\|^{2} \\
& -\alpha_{2}^{k+1} \alpha_{3}^{k+1} \prod_{j=1}^{k}\left(1-\alpha_{3}^{j}\right)\left\|U_{n, k+2} x_{0}-x_{0}\right\|^{2} \\
& -\alpha_{1}^{k} \alpha_{2}^{k} \prod_{j=1}^{k-1}\left(1-\alpha_{3}^{j}\right)\left\|T_{k} U_{n, k+1} x_{0}-U_{n, k+1} x_{0}\right\|^{2} \\
& -\alpha_{2}^{k} \alpha_{3}^{k} \prod_{j=1}^{k-1}\left(1-\alpha_{3}^{j}\right)\left\|U_{n, k+1} x_{0}-x_{0}\right\|^{2} \\
& -\alpha_{1}^{3} \alpha_{2}^{3} \prod_{j=1}^{2}\left(1-\alpha_{3}^{j}\right)\left\|T_{3} U_{n, 4} x_{0}-U_{n, 4} x_{0}\right\|^{2}-\alpha_{2}^{3} \alpha_{3}^{3} \prod_{j=1}^{2}\left(1-\alpha_{3}^{j}\right)\left\|U_{n, 4} x_{0}-x_{0}\right\|^{2} \\
& -\alpha_{1}^{1} \alpha_{2}^{1}\left\|T_{1} U_{n, 2} x_{0}-U_{n, 2} x_{0}^{k-1} \prod_{j=1}^{k-2}\left(1-\alpha_{3}^{j}\right)\right\| T_{k-1} U_{n, k} x_{0}-\alpha_{2}^{1} \alpha_{3}^{1}\left\|U_{n, 2} x_{0}-x_{0}\right\|^{2} . \\
& \vdots \\
& -\alpha_{2}^{k-1} \alpha_{3}^{k-1} \prod_{j=1}^{k-2}\left(1-\alpha_{3}^{j}\right)\left\|U_{n, k} x_{0}-x_{0}\right\|^{2} \\
& \\
&
\end{aligned}
$$

For $k \in \mathbb{N}$ and (2.20), we have

$$
\alpha_{2}^{k-1} \alpha_{3}^{k-1} \prod_{j=1}^{k-2}\left(1-\alpha_{3}^{j}\right)\left\|U_{n, k} x_{0}-x_{0}\right\|^{2} \leq\left\|x_{0}-x^{*}\right\|^{2}-\left\|S_{n} x_{0}-x^{*}\right\|^{2}
$$

as $n \rightarrow \infty$. This implies that $U_{\infty, k} x_{0}=x_{0}$, for all $k \in \mathbb{N}$. 
Abstract and Applied Analysis

Again by (2.20), we have

$$
\alpha_{1}^{k} \alpha_{2}^{k} \prod_{j=1}^{k-1}\left(1-\alpha_{3}^{j}\right)\left\|T_{k} U_{n, k+1} x_{0}-U_{n, k+1} x_{0}\right\|^{2} \leq\left\|x_{0}-x^{*}\right\|^{2}-\left\|S_{n} x_{0}-x^{*}\right\|^{2}
$$

as $n \rightarrow \infty$. Hence,

$$
\alpha_{1}^{k} \alpha_{2}^{k} \prod_{j=1}^{k-1}\left(1-\alpha_{3}^{j}\right)\left\|T_{k} U_{\infty, k+1} x_{0}-U_{\infty, k+1} x_{0}\right\|^{2} \leq 0 .
$$

From $U_{\infty, k} x_{0}=x_{0}$, for all $k \in \mathbb{N}$, and (2.23), we obtain that $T_{k} x_{0}=x_{0}$, for all $k \in \mathbb{N}$. This implies that $x_{0} \in \bigcap_{i=1}^{\infty} F\left(T_{i}\right)$.

\section{Main Result}

Theorem 3.1. Let $C$ be a nonempty closed convex subset of a Hilbert space $H$. Let $F$ be bifunctions from $C \times C$ into $\mathbb{R}$ satisfying (A1)-(A4). Let $A: C \rightarrow H$ be a $\alpha$-inverse-strongly monotone mapping. Let $\left\{T_{i}\right\}_{i=1}^{\infty}$ be infinite family of nonexpansive mappings with $\mathfrak{F}=\bigcap_{i=1}^{\infty} F\left(T_{i}\right) \bigcap \operatorname{EP}(F) \bigcap \operatorname{VI}(C, A)$, and let $\rho_{j}=\left(\alpha_{1}^{j}, \alpha_{2}^{j}, \alpha_{3}^{j}\right) \in I \times I \times I$, where $I=[0,1], \alpha_{1}^{j}+\alpha_{2}^{j}+\alpha_{3}^{j}=1, \alpha_{1}^{j}+\alpha_{2}^{j} \leq b<1$, and $\alpha_{1}^{j}, \alpha_{2}^{j}, \alpha_{3}^{j} \in(0,1)$ for all $j=1,2, \ldots$. For every $n \in \mathbb{N}$, let $S_{n}$ and $S$ be $S$-mappings generated by $T_{n}, \ldots, T_{1}$ and $\rho_{n}, \rho_{n-1}, \ldots, \rho_{1}$ and $T_{n}, T_{n-1}, \ldots$, and $\rho_{n}, \rho_{n-1}, \ldots$, respectively. Let $\left\{x_{n}\right\},\left\{u_{n}\right\}$ be sequences generated by $x_{1}, u \in C$ and

$$
\begin{gathered}
F\left(u_{n}, y\right)+\frac{1}{r_{n}}\left\langle y-u_{n}, u_{n}-x_{n}\right\rangle \geq 0, \quad \forall y \in C, \\
x_{n+1}=\alpha_{n} u+\beta_{n} P_{C}(I-\lambda A) x_{n}+\gamma_{n} S_{n} P_{C}(I-\lambda A) u_{n}, \quad \forall n \geq 1,
\end{gathered}
$$

where $\left\{\alpha_{n}\right\},\left\{\beta_{n}\right\},\left\{\gamma_{n}\right\} \in(0,1)$, such that $\alpha_{n}+\beta_{n}+\gamma_{n}=1, \beta_{n} \in[c, d] \subset(0,1) r_{n} \in[a, b] \subset$ $(0,2 \alpha), \lambda \subset(0,2 \alpha)$. Assume that

(i) $\lim _{n \rightarrow \infty} \alpha_{n}=0$ and $\sum_{n=0}^{\infty} \alpha_{n}=\infty$,

(ii) $\sum_{n=1}^{\infty} \alpha_{1}^{n}<\infty$,

(iii) $\sum_{n=1}^{\infty}\left|r_{n+1}-r_{n}\right|, \sum_{n=1}^{\infty}\left|\gamma_{n+1}-\gamma_{n}\right|, \sum_{n=1}^{\infty}\left|\alpha_{n+1}-\alpha_{n}\right|, \sum_{n=1}^{\infty}\left|\beta_{n+1}-\beta_{n}\right|<\infty$,

then the sequence $\left\{x_{n}\right\},\left\{u_{n}\right\}$ converge strongly to $z=P_{\mathfrak{F}} u$. 
Proof. First, we show that $(I-\lambda A)$ is nonexpansive. Let $x, y \in C$. Since $A$ is $\alpha$-inverse-strongly monotone and $\lambda<2 \alpha$, we have

$$
\begin{aligned}
\|(I-\lambda A) x-(I-\lambda A) y\|^{2} & =\|x-y-\lambda(A x-A y)\|^{2} \\
& =\|x-y\|^{2}-2 \lambda\langle x-y, A x-A y\rangle+\lambda^{2}\|A x-A y\|^{2} \\
& \leq\|x-y\|^{2}-2 \alpha \lambda\|A x-A y\|^{2}+\lambda^{2}\|A x-A y\|^{2} \\
& =\|x-y\|^{2}+\lambda(\lambda-2 \alpha)\|A x-A y\|^{2} \\
& \leq\|x-y\|^{2} .
\end{aligned}
$$

Thus, $(I-\lambda A)$ is nonexpansive. We will divide our proof into 5 steps.

Step 1. We shall show that the sequence $\left\{x_{n}\right\}$ is bounded. Since

$$
F\left(u_{n}, y\right)+\frac{1}{r_{n}}\left\langle y-u_{n}, u_{n}-x_{n}\right\rangle \geq 0, \quad \forall y \in C .
$$

By Lemma 2.5, we have $u_{n}=T_{r_{n}} x_{n}$ and $\mathrm{EP}(F)=F\left(T_{r_{n}}\right)$.

Let $z \in \mathfrak{F}$. By nonexpansiveness of $I-\lambda A$ and $T_{r_{n}}$, we have

$$
\begin{aligned}
\left\|x_{n+1}-z\right\| & =\left\|\alpha_{n} u+\beta_{n} P_{C}(I-\lambda A) x_{n}+\gamma_{n} S_{n} P_{C}(I-\lambda A) u_{n}-z\right\| \\
& =\left\|\alpha_{n}(u-z)+\beta_{n}\left(P_{C}(I-\lambda A) x_{n}-z\right)+\gamma_{n}\left(S_{n} P_{C}(I-\lambda A) u_{n}-z\right)\right\| \\
& \leq \alpha_{n}\|u-z\|+\beta_{n}\left\|P_{C}(I-\lambda A) x_{n}-z\right\|+\gamma_{n}\left\|S_{n} P_{C}(I-\lambda A) u_{n}-z\right\| \\
& \leq \alpha_{n}\|u-z\|+\beta_{n}\left\|x_{n}-z\right\|+\gamma_{n}\left\|u_{n}-z\right\| \\
& =\alpha_{n}\|u-z\|+\beta_{n}\left\|x_{n}-z\right\|+\gamma_{n}\left\|T_{r_{n}} x_{n}-z\right\| \\
& \leq \alpha_{n}\|u-z\|+\left(1-\alpha_{n}\right)\left\|x_{n}-z\right\| \\
& \leq \max \left\{\|u-z\|,\left\|x_{n}-z\right\|\right\} .
\end{aligned}
$$

By induction, we can prove that $\left\{x_{n}\right\}$ is bounded and so is $\left\{u_{n}\right\}$. 
Step 2. We will show that $\lim _{n \rightarrow \infty}\left\|x_{n+1}-x_{n}\right\|=0$. By definition of $x_{n}$, we have

$$
\begin{aligned}
\left\|x_{n+1}-x_{n}\right\|= & \| \alpha_{n} u+\beta_{n} P_{C}(I-\lambda A) x_{n}+\gamma_{n} S_{n} P_{C}(I-\lambda A) u_{n} \\
& -\alpha_{n-1} u-\beta_{n-1} P_{C}(I-\lambda A) x_{n-1}-\gamma_{n-1} S_{n-1} P_{C}(I-\lambda A) u_{n-1} \| \\
= & \|\left(\alpha_{n}-\alpha_{n-1}\right) u+\beta_{n} P_{C}(I-\lambda A) x_{n}-\beta_{n-1} P_{C}(I-\lambda A) x_{n}+\beta_{n-1} P_{C}(I-\lambda A) x_{n} \\
& +\gamma_{n} S_{n} P_{C}(I-\lambda A) u_{n}-\gamma_{n-1} S_{n} P_{C}(I-\lambda A) u_{n}+\gamma_{n-1} S_{n} P_{C}(I-\lambda A) u_{n} \\
& \quad-\beta_{n-1} P_{C}(I-\lambda A) x_{n-1}-\gamma_{n-1} S_{n-1} P_{C}(I-\lambda A) u_{n-1} \| \\
= & \|\left(\alpha_{n}-\alpha_{n-1}\right) u+\left(\beta_{n}-\beta_{n-1}\right) P_{C}(I-\lambda A) x_{n} \\
& \quad+\beta_{n-1}\left(P_{C}(I-\lambda A) x_{n}-P_{C}(I-\lambda A) x_{n-1}\right)+\left(\gamma_{n}-\gamma_{n-1}\right) S_{n} P_{C}(I-\lambda A) u_{n} \\
& +\gamma_{n-1}\left(S_{n} P_{C}(I-\lambda A) u_{n}-S_{n-1} P_{C}(I-\lambda A) u_{n-1}\right) \| \\
\leq & \left|\alpha_{n}-\alpha_{n-1}\right|\|u\|+\left|\beta_{n}-\beta_{n-1}\right|\left\|P_{C}(I-\lambda A) x_{n}\right\| \\
& +\beta_{n-1}\left\|P_{C}(I-\lambda A) x_{n}-P_{C}(I-\lambda A) x_{n-1}\right\|+\left|\gamma_{n}-\gamma_{n-1}\right|\left\|S_{n} P_{C}(I-\lambda A) u_{n}\right\| \\
& +\gamma_{n-1}\left\|S_{n} P_{C}(I-\lambda A) u_{n}-S_{n-1} P_{C}(I-\lambda A) u_{n-1}\right\| \\
\leq \mid & \alpha_{n}-\alpha_{n-1}|\|u\|+| \beta_{n}-\beta_{n-1} \mid\left\|P_{C}(I-\lambda A) x_{n}\right\| \\
& +\beta_{n-1}\left\|x_{n}-x_{n-1}\right\|+\left|\gamma_{n}-\gamma_{n-1}\right|\left\|S_{n} P_{C}(I-\lambda A) u_{n}\right\| \\
& +\gamma_{n-1}\left(\left\|S_{n} P_{C}(I-\lambda A) u_{n}-S_{n} P_{C}(I-\lambda A) u_{n-1}\right\|\right. \\
& \left.\quad+\left\|S_{n} P_{C}(I-\lambda A) u_{n-1}-S_{n-1} P_{C}(I-\lambda A) u_{n-1}\right\|\right) \\
\leq & \left|\alpha_{n}-\alpha_{n-1}\right|\|u\|+\left|\beta_{n}-\beta_{n-1}\right|\left\|P_{C}(I-\lambda A) x_{n}\right\| \\
+ & \beta_{n-1}\left\|x_{n}-x_{n-1}\right\|+\left|\gamma_{n}-\gamma_{n-1}\right|\left\|S_{n} P_{C}(I-\lambda A) u_{n}\right\| \\
+ & \gamma_{n-1}\left\|u_{n}-u_{n-1}\right\|+\gamma_{n-1}\left\|S_{n} P_{C}(I-\lambda A) u_{n-1}-S_{n-1} P_{C}(I-\lambda A) u_{n-1}\right\| .
\end{aligned}
$$

Since $u_{n}=T_{r_{n}} x_{n}$, by definition of $T_{r_{n}}$, we have

$$
F\left(T_{r_{n}} x_{n}, y\right)+\frac{1}{r_{n}}\left\langle y-T_{r_{n}} x_{n}, T_{r_{n}} x_{n}-x_{n}\right\rangle \geq 0, \quad \forall y \in C .
$$

Similarly,

$$
F\left(T_{r_{n+1}} x_{n+1}, y\right)+\frac{1}{r_{n+1}}\left\langle y-T_{r_{n+1}} x_{n+1}, T_{r_{n+1}} x_{n+1}-x_{n+1}\right\rangle \geq 0, \quad \forall y \in C .
$$


From (3.6) and (3.7), we obtain

$$
\begin{gathered}
F\left(T_{r_{n}} x_{n}, T_{r_{n+1}} x_{n+1}\right)+\frac{1}{r_{n}}\left\langle T_{r_{n+1}} x_{n+1}-T_{r_{n}} x_{n}, T_{r_{n}} x_{n}-x_{n}\right\rangle \geq 0, \\
F\left(T_{r_{n+1}} x_{n+1}, T_{r_{n}} x_{n}\right)+\frac{1}{r_{n+1}}\left\langle T_{r_{n}} x_{n}-T_{r_{n+1}} x_{n+1}, T_{r_{n+1}} x_{n+1}-x_{n+1}\right\rangle \geq 0 .
\end{gathered}
$$

By (3.8), we have

$$
\frac{1}{r_{n}}\left\langle T_{r_{n+1}} x_{n+1}-T_{r_{n}} x_{n}, T_{r_{n}} x_{n}-x_{n}\right\rangle+\frac{1}{r_{n+1}}\left\langle T_{r_{n}} x_{n}-T_{r_{n+1}} x_{n+1}, T_{r_{n+1}} x_{n+1}-x_{n+1}\right\rangle \geq 0 .
$$

It follows that

$$
\left\langle T_{r_{n}} x_{n}-T_{r_{n+1}} x_{n+1}, \frac{T_{r_{n+1}} x_{n+1}-x_{n+1}}{r_{n+1}}-\frac{T_{r_{n}} x_{n}-x_{n}}{r_{n}}\right\rangle \geq 0 .
$$

This implies that

$$
0 \leq\left\langle T_{r_{n+1}} x_{n+1}-T_{r_{n}} x_{n}, T_{r_{n}} x_{n}-T_{r_{n+1}} x_{n+1}+T_{r_{n+1}} x_{n+1}-x_{n}-\frac{r_{n}}{r_{n+1}}\left(T_{r_{n+1}} x_{n+1}-x_{n+1}\right)\right\rangle .
$$

It follows that

$$
\begin{aligned}
\left\|T_{r_{n+1}} x_{n+1}-T_{r_{n}} x_{n}\right\|^{2} & \leq\left\langle T_{r_{n+1}} x_{n+1}-T_{r_{n}} x_{n}, T_{r_{n+1}} x_{n+1}-x_{n}-\frac{r_{n}}{r_{n+1}}\left(T_{r_{n+1}} x_{n+1}-x_{n+1}\right)\right\rangle \\
& =\left\langle T_{r_{n+1}} x_{n+1}-T_{r_{n}} x_{n}, x_{n+1}-x_{n}+\left(1-\frac{r_{n}}{r_{n+1}}\right)\left(T_{r_{n+1}} x_{n+1}-x_{n+1}\right)\right\rangle \\
& \leq\left\|T_{r_{n+1}} x_{n+1}-T_{r_{n}} x_{n}\right\|\left\|x_{n+1}-x_{n}+\left(1-\frac{r_{n}}{r_{n+1}}\right)\left(T_{r_{n+1}} x_{n+1}-x_{n+1}\right)\right\| \\
& \leq\left\|T_{r_{n+1}} x_{n+1}-T_{r_{n}} x_{n}\right\|\left(\left\|x_{n+1}-x_{n}\right\|+\left|1-\frac{r_{n}}{r_{n+1}}\right|\left\|T_{r_{n+1}} x_{n+1}-x_{n+1}\right\|\right) \\
& =\left\|T_{r_{n+1}} x_{n+1}-T_{r_{n}} x_{n}\right\|\left(\left\|x_{n+1}-x_{n}\right\|+\frac{1}{r_{n+1}}\left|r_{n+1}-r_{n}\right|\left\|T_{r_{n+1}} x_{n+1}-x_{n+1}\right\|\right) \\
& \leq\left\|T_{r_{n+1}} x_{n+1}-T_{r_{n}} x_{n}\right\|\left(\left\|x_{n+1}-x_{n}\right\|+\frac{1}{a}\left|r_{n+1}-r_{n}\right|\left\|T_{r_{n+1}} x_{n+1}-x_{n+1}\right\|\right) .
\end{aligned}
$$

It follows that

$$
\left\|u_{n+1}-u_{n}\right\| \leq\left\|x_{n+1}-x_{n}\right\|+\frac{1}{a}\left|r_{n+1}-r_{n}\right|\left\|u_{n+1}-x_{n+1}\right\| .
$$


Putting $y_{n}=P_{C}(I-\lambda A) u_{n}$, then $\left\{y_{n}\right\}$ is bounded. By definition of $S_{n}$, for all $n \in \mathbb{N}$, we have

$$
\begin{aligned}
\left\|S_{n} y_{n-1}-S_{n-1} y_{n-1}\right\|= & \left\|U_{n, 1} y_{n-1}-U_{n-1,1} y_{n-1}\right\| \\
= & \| \alpha_{1}^{1} T_{1} U_{n, 2} y_{n-1}+\alpha_{2}^{1} U_{n, 2} y_{n-1}+\alpha_{3}^{1} y_{n-1} \\
& -\alpha_{1}^{1} T_{1} U_{n-1,2} y_{n-1}-\alpha_{2}^{1} U_{n-1,2} y_{n-1}-\alpha_{3}^{1} y_{n-1} \| \\
\leq & \left(1-\alpha_{3}^{1}\right)\left\|U_{n, 2} y_{n-1}-U_{n-1,2} y_{n-1}\right\| \\
\leq & \left(1-\alpha_{3}^{1}\right)\left(1-\alpha_{3}^{2}\right)\left\|U_{n, 3} y_{n-1}-U_{n-1,3} y_{n-1}\right\| \\
= & \prod_{j=1}^{2}\left(1-\alpha_{3}^{j}\right)\left\|U_{n, 3} y_{n-1}-U_{n-1,3} y_{n-1}\right\| \\
& \vdots \\
\leq & \prod_{j=1}^{n-1}\left(1-\alpha_{3}^{j}\right)\left\|U_{n, n} y_{n-1}-U_{n-1, n} y_{n-1}\right\| \\
\leq & \left\|U_{n, n} y_{n-1}-y_{n-1}\right\| \\
= & \left\|\alpha_{1}^{n} T_{n} y_{n-1}+\left(1-\alpha_{1}^{n}\right) y_{n-1}-y_{n-1}\right\| \\
= & \alpha_{1}^{n}\left\|T_{n} y_{n-1}-y_{n-1}\right\| \\
\leq & \alpha_{1}^{n} 2\left\|y_{n-1}-z\right\| .
\end{aligned}
$$

Substituting (3.13) and (3.14) into (3.5), we have

$$
\begin{aligned}
\left\|x_{n+1}-x_{n}\right\| \leq & \left|\alpha_{n}-\alpha_{n-1}\right|\|u\|+\left|\beta_{n}-\beta_{n-1}\right|\left\|P_{C}(I-\lambda A) x_{n}\right\| \\
& +\beta_{n-1}\left\|x_{n}-x_{n-1}\right\|+\left|\gamma_{n}-\gamma_{n-1}\right|\left\|S_{n} P_{C}(I-\lambda A) u_{n}\right\| \\
& +\gamma_{n-1}\left\|u_{n}-u_{n-1}\right\|+\gamma_{n-1}\left\|S_{n} P_{C}(I-\lambda A) u_{n-1}-S_{n-1} P_{C}(I-\lambda A) u_{n-1}\right\| \\
\leq & \left|\alpha_{n}-\alpha_{n-1}\right|\|u\|+\left|\beta_{n}-\beta_{n-1}\right|\left\|P_{C}(I-\lambda A) x_{n}\right\| \\
& +\beta_{n-1}\left\|x_{n}-x_{n-1}\right\|+\left|\gamma_{n}-\gamma_{n-1}\right|\left\|S_{n} P_{C}(I-\lambda A) u_{n}\right\| \\
& +\gamma_{n-1}\left(\left\|x_{n+1}-x_{n}\right\|+\frac{1}{a}\left|r_{n+1}-r_{n}\right|\left\|u_{n+1}-x_{n+1}\right\|\right) \\
& +2 \gamma_{n-1} \alpha_{1}^{n}\left\|y_{n-1}-z\right\| \\
\leq & \left|\alpha_{n}-\alpha_{n-1}\right|\|u\|+\left|\beta_{n}-\beta_{n-1}\right|\left\|P_{C}(I-\lambda A) x_{n}\right\| \\
& +\left(1-\alpha_{n-1}\right)\left\|x_{n}-x_{n-1}\right\|+\left|\gamma_{n}-\gamma_{n-1}\right|\left\|S_{n} P_{C}(I-\lambda A) u_{n}\right\| \\
& +\frac{1}{a}\left|r_{n+1}-r_{n}\right| \mid u_{n+1}-x_{n+1}\left\|+2 \gamma_{n-1} \alpha_{1}^{n}\right\| y_{n-1}-z \| \\
\leq & \left(1-\alpha_{n-1}\right)\left\|x_{n}-x_{n-1}\right\|+\left|\beta_{n}-\beta_{n-1}\right| M_{1} \\
& +\left|\alpha_{n}-\alpha_{n-1}\right| M_{1}+\left|\gamma_{n}-\gamma_{n-1}\right| M_{1}+\frac{1}{a}\left|r_{n+1}-r_{n}\right| M_{1}+2 \alpha_{1}^{n} M_{1}
\end{aligned}
$$


where $M_{1}=\max _{n \in \mathbb{N}}\left\{\|u\|,\left\|P_{C}(I-\lambda A) x_{n}\right\|,\left\|S_{n} P_{C}(I-\lambda A) u_{n}\right\|,\left\|u_{n}-x_{n}\right\|,\left\|y_{n}-z\right\|\right\}$. By (3.15), Lemma 2.3, and conditions (i)-(iii), we obtain

$$
\lim _{n \rightarrow \infty}\left\|x_{n+1}-x_{n}\right\|=0
$$

Step 3. We shall show that $\lim _{n \rightarrow \infty}\left\|x_{n}-u_{n}\right\|=0$.

Let $v \in \mathfrak{F}$. Since $u_{n}=T_{r_{n}} x_{n}$ and $T_{r_{n}}$ is firmly nonexpansive, we have

$$
\begin{aligned}
\left\|v-T_{r_{n}} x_{n}\right\|^{2} & =\left\|T_{r_{n}} v-T_{r_{n}} x_{n}\right\|^{2} \\
& \leq\left\langle T_{r_{n}} v-T_{r_{n}} x_{n}, v-x_{n}\right\rangle \\
& =\frac{1}{2}\left(\left\|T_{r_{n}} x_{n}-v\right\|^{2}+\left\|x_{n}-v\right\|^{2}-\left\|T_{r_{n}} x_{n}-x_{n}\right\|^{2}\right) .
\end{aligned}
$$

Hence,

$$
\left\|u_{n}-v\right\|^{2} \leq\left\|x_{n}-v\right\|^{2}-\left\|u_{n}-x_{n}\right\|^{2}
$$

By (3.18), we have

$$
\begin{aligned}
\left\|x_{n+1}-v\right\|^{2} & =\left\|\alpha_{n}(u-v)+\beta_{n}\left(P_{C}(I-\lambda A) x_{n}-v\right)+\gamma_{n}\left(S_{n} P_{C}(I-\lambda A) u_{n}-v\right)\right\|^{2} \\
& \leq \alpha_{n}\|u-v\|^{2}+\beta_{n}\left\|x_{n}-v\right\|^{2}+\gamma_{n}\left\|u_{n}-v\right\|^{2} \\
& \leq \alpha_{n}\|u-v\|^{2}+\beta_{n}\left\|x_{n}-v\right\|^{2}+\gamma_{n}\left(\left\|x_{n}-v\right\|^{2}-\left\|u_{n}-x_{n}\right\|^{2}\right) \\
& \leq \alpha_{n}\|u-v\|^{2}+\left\|x_{n}-v\right\|^{2}-\gamma_{n}\left\|u_{n}-x_{n}\right\|^{2}
\end{aligned}
$$

it implies that

$$
\begin{aligned}
\gamma_{n}\left\|u_{n}-x_{n}\right\|^{2} & \leq \alpha_{n}\|u-v\|^{2}+\left\|x_{n}-v\right\|^{2}-\left\|x_{n+1}-v\right\|^{2} \\
& \leq \alpha_{n}\|u-v\|^{2}+\left(\left\|x_{n}-v\right\|-\left\|x_{n+1}-v\right\|\right)\left(\left\|x_{n}-v\right\|+\left\|x_{n+1}-v\right\|\right) \\
& \leq \alpha_{n}\|u-v\|^{2}+\left\|x_{n}-x_{n+1}\right\|\left(\left\|x_{n}-v\right\|+\left\|x_{n+1}-v\right\|\right) .
\end{aligned}
$$

By (3.16) and condition (i), we have

$$
\lim _{n \rightarrow \infty}\left\|x_{n}-u_{n}\right\|=0
$$


Let $z \in \mathfrak{F}$ and by nonexpansiveness of $I-\lambda A$, we have

$$
\begin{aligned}
\left\|x_{n+1}-z\right\|^{2} \leq & \alpha_{n}\|u-z\|^{2}+\beta_{n}\left\|P_{C}(I-\lambda A) x_{n}-z\right\|^{2}+\gamma_{n}\left\|S_{n} P_{C}(I-\lambda A) u_{n}-z\right\|^{2} \\
& -\beta_{n} \gamma_{n}\left\|P_{C}(I-\lambda A) x_{n}-S_{n} P_{C}(I-\lambda A) u_{n}\right\| \\
\leq & \alpha_{n}\|u-z\|^{2}+\left(1-\alpha_{n}\right)\left\|x_{n}-z\right\|^{2} \\
& -\beta_{n} \gamma_{n}\left\|P_{C}(I-\lambda A) x_{n}-S_{n} P_{C}(I-\lambda A) u_{n}\right\| \\
\leq & \alpha_{n}\|u-z\|^{2}+\left\|x_{n}-z\right\|^{2} \\
& -\beta_{n} \gamma_{n}\left\|P_{C}(I-\lambda A) x_{n}-S_{n} P_{C}(I-\lambda A) u_{n}\right\| .
\end{aligned}
$$

It implies that

$$
\begin{aligned}
& \beta_{n} \gamma_{n}\left\|P_{C}(I-\lambda A) x_{n}-S_{n} P_{C}(I-\lambda A) u_{n}\right\| \\
& \leq \alpha_{n}\|u-z\|^{2}+\left\|x_{n}-z\right\|^{2}-\left\|x_{n+1}-z\right\|^{2} \\
& =\alpha_{n}\|u-z\|^{2}+\left(\left\|x_{n}-z\right\|-\left\|x_{n+1}-z\right\|\right)\left(\left\|x_{n}-z\right\|+\left\|x_{n+1}-z\right\|\right) \\
& =\alpha_{n}\|u-z\|^{2}+\left\|x_{n}-x_{n+1}\right\|\left(\left\|x_{n}-z\right\|+\left\|x_{n+1}-z\right\|\right) .
\end{aligned}
$$

By (3.16) and condition (i), we have

$$
\lim _{n \rightarrow \infty}\left\|P_{C}(I-\lambda A) x_{n}-S_{n} P_{C}(I-\lambda A) u_{n}\right\|=0 .
$$

Since

$$
\begin{aligned}
\left\|P_{C}(I-\lambda A) x_{n}-S_{n} P_{C}(I-\lambda A) x_{n}\right\| \leq & \left\|P_{C}(I-\lambda A) x_{n}-S_{n} P_{C}(I-\lambda A) u_{n}\right\| \\
& +\left\|S_{n} P_{C}(I-\lambda A) u_{n}-S_{n} P_{C}(I-\lambda A) x_{n}\right\| \\
\leq & \left\|P_{C}(I-\lambda A) x_{n}-S_{n} P_{C}(I-\lambda A) u_{n}\right\|+\left\|u_{n}-x_{n}\right\|,
\end{aligned}
$$

by (3.24) and (3.21), we have

$$
\lim _{n \rightarrow \infty}\left\|P_{C}(I-\lambda A) x_{n}-S_{n} P_{C}(I-\lambda A) x_{n}\right\|=0 .
$$

Since

$$
\begin{aligned}
\left\|x_{n}-P_{C}(I-\lambda A) x_{n}\right\| \leq & \left\|x_{n}-x_{n+1}\right\|+\left\|x_{n+1}-P_{C}(I-\lambda A) x_{n}\right\| \\
\leq & \left\|x_{n}-x_{n+1}\right\|+\alpha_{n}\left\|u-P_{C}(I-\lambda A) x_{n}\right\| \\
& +\gamma_{n}\left\|S_{n} P_{C}(I-\lambda A) u_{n}-P_{C}(I-\lambda A) x_{n}\right\|,
\end{aligned}
$$


by (3.24), (3.16), and condition (i), we have

$$
\lim _{n \rightarrow \infty}\left\|x_{n}-P_{C}(I-\lambda A) x_{n}\right\|=0 .
$$

Since

$$
\begin{aligned}
\left\|x_{n}-S_{n} P_{C}(I-\lambda A) u_{n}\right\| \leq & \left\|x_{n}-x_{n+1}\right\|+\left\|x_{n+1}-S_{n} P_{C}(I-\lambda A) u_{n}\right\| \\
\leq & \left\|x_{n}-x_{n+1}\right\|+\alpha_{n}\left\|u-S_{n} P_{C}(I-\lambda A) u_{n}\right\| \\
& +\beta_{n}\left\|P_{C}(I-\lambda A) x_{n}-S_{n} P_{C}(I-\lambda A) u_{n}\right\|,
\end{aligned}
$$

again by (3.24), (3.16), and condition (i), we have

$$
\lim _{n \rightarrow \infty}\left\|x_{n}-S_{n} P_{C}(I-\lambda A) u_{n}\right\|=0
$$

Since

$$
\begin{aligned}
\left\|x_{n}-S_{n} P_{C}(I-\lambda A) x_{n}\right\| & \leq\left\|x_{n}-S_{n} P_{C}(I-\lambda A) u_{n}\right\|+\left\|S_{n} P_{C}(I-\lambda A) u_{n}-S_{n} P_{C}(I-\lambda A) x_{n}\right\| \\
& \leq\left\|x_{n}-S_{n} P_{C}(I-\lambda A) u_{n}\right\|+\left\|u_{n}-x_{n}\right\|,
\end{aligned}
$$

by (3.21) and (3.30), we have

$$
\lim _{n \rightarrow \infty}\left\|x_{n}-S_{n} P_{C}(I-\lambda A) x_{n}\right\|=0
$$

Step 4. Putting $z_{0}=P_{\mathfrak{F}} u$, we will show that

$$
\limsup _{n \rightarrow \infty}\left\langle u-z_{0}, x_{n}-z_{0}\right\rangle \leq 0
$$

To show this inequality, take a subsequence $\left\{x_{n_{m}}\right\}$ of $\left\{x_{n}\right\}$, such that

$$
\limsup _{n \rightarrow \infty}\left\langle u-z_{0}, x_{n}-z_{0}\right\rangle=\limsup _{m \rightarrow \infty}\left\langle u-z_{0}, x_{n_{m}}-z_{0}\right\rangle
$$

Without loss of generality, we may assume that $x_{n_{m}} \rightarrow \omega$ as $m \rightarrow \infty$ where $\omega \in C$. By nonexpansiveness of $P_{C}(I-\lambda A)$, (3.28), and Lemma 2.2, we have $\omega \in F\left(P_{C}(I-\lambda A)\right)$. By Lemma 2.6, we obtain that $\omega \in \operatorname{VI}(C, A)$. Since $\left\|u_{n_{m}}-x_{n_{m}}\right\| \rightarrow 0$ as $m \rightarrow \infty$, we have $u_{n_{m}} \rightarrow \omega$ as $m \rightarrow \infty$. Since

$$
F\left(u_{n}, y\right)+\frac{1}{r_{n}}\left\langle y-u_{n}, u_{n}-x_{n}\right\rangle \geq 0, \quad \forall y \in C .
$$


By (A2), we have

$$
\frac{1}{r_{n}}\left\langle y-u_{n}, u_{n}-x_{n}\right\rangle \geq F\left(y, u_{n}\right), \quad \forall y \in C
$$

In particular,

$$
\left\langle y-u_{n_{m}}, \frac{1}{r_{n_{m}}}\left(u_{n_{m}}-x_{n_{m}}\right)\right\rangle \geq F\left(y, u_{n_{m}}\right) .
$$

By condition (A4), $F(y, \cdot)$ is lower semicontinuous and convex, and thus weakly semicontinuous. By (3.21) imply that $\left(1 / r_{n_{m}}\right)\left(u_{n_{m}}-x_{n_{m}}\right) \rightarrow 0$ in norm. Therefore, letting $m \rightarrow \infty$ in (3.37), we have

$$
F(y, \omega) \leq \lim _{m \rightarrow \infty} F\left(y, u_{n_{m}}\right) \leq 0, \quad \forall y \in C
$$

Replacing $y$ with $y_{t}:=t y+(1-t) \omega, t \in(0,1]$, we have $y_{t} \in C$, and using (A1), (A4), and (3.38), we obtain

$$
0=F\left(y_{t}, y_{t}\right) \leq t F\left(y_{t}, y\right)+(1-t) F\left(y_{t}, \omega\right) \leq t F\left(y_{t}, y\right)
$$

Hence, $F(t y+(1-t) \omega, y) \geq 0$, for all $t \in(0,1]$ and for all $y \in C$. Letting $t \rightarrow 0^{+}$and using assumption (A3), we can conclude that

$$
F(\omega, y) \geq 0, y \in C
$$

Therefore, $\omega \in \operatorname{EP}(F)$.

We will show that $\omega \in \bigcap_{i=1}^{\infty} F\left(T_{i}\right)$. By Lemma 2.10, we have $F(S)=\bigcap_{i=1}^{\infty} F\left(T_{i}\right)$. Assume that $\omega \neq S \omega$. Using Opial s' property, (3.32), $\omega \in F\left(P_{C}(I-\lambda A)\right)$, and Remark 2.9, we have

$$
\begin{aligned}
\liminf _{m \rightarrow \infty}\left\|x_{n_{m}}-\omega\right\|<\liminf _{m \rightarrow \infty} & \left\|x_{n_{m}}-S \omega\right\| \\
\leq \liminf _{m \rightarrow \infty} & \left\|x_{n_{m}}-S_{n_{m}} P_{C}(I-\lambda A) x_{n_{m}}\right\| \\
& +\left\|S_{n_{m}} P_{C}(I-\lambda A) x_{n_{m}}-S_{n_{m}} P_{C}(I-\lambda A) \omega\right\| \\
& \left.+\left\|S_{n_{m}} P_{C}(I-\lambda A) \omega-S \omega\right\|\right) \\
=\liminf _{m \rightarrow \infty} & \left(\left\|x_{n_{m}}-S_{n_{m}} P_{C}(I-\lambda A) x_{n_{m}}\right\|\right. \\
& +\left\|S_{n_{m}} P_{C}(I-\lambda A) x_{n_{m}}-S_{n_{m}} P_{C}(I-\lambda A) \omega\right\| \\
& \left.+\left\|S_{n_{m}} \omega-S \omega\right\|\right) \\
\leq \liminf _{m \rightarrow \infty} & \left\|x_{n_{m}}-\omega\right\| .
\end{aligned}
$$

This is a contradiction, then $\omega \in \bigcap_{i=1}^{\infty} F\left(T_{i}\right)$. Hence, $\omega \in \mathfrak{F}$. 
Since $x_{n_{m}} \rightarrow \omega$ and $\omega \in \mathfrak{F}$, we have

$$
\limsup _{n \rightarrow \infty}\left\langle u-z_{0}, x_{n}-z_{0}\right\rangle=\limsup _{m \rightarrow \infty}\left\langle u-z_{0}, x_{n_{m}}-z_{0}\right\rangle=\left\langle u-z_{0}, \omega-z_{0}\right\rangle \leq 0 .
$$

Step 5. Finally, we show that $\left\{x_{n}\right\}$ and $\left\{u_{n}\right\}$ converse strongly to $z_{0}=P_{\mathfrak{F}} u$. Putting $z_{0}=P_{\mathfrak{F}} u$, by nonexpansiveness of $P_{C}(I-\lambda A), S_{n}$, and $T_{r_{n}}$, we have

$$
\begin{aligned}
\left\|x_{n+1}-z_{0}\right\|^{2} & =\left\|\alpha_{n}\left(u-z_{0}\right)+\beta_{n}\left(P_{C}(I-\lambda A) x_{n}-z_{0}\right)+\gamma_{n}\left(S_{n} P_{C}(I-\lambda A) u_{n}-z_{0}\right)\right\|^{2} \\
& \leq\left\|\beta_{n}\left(P_{C}(I-\lambda A) x_{n}-z_{0}\right)+\gamma_{n}\left(S_{n} P_{C}(I-\lambda A) u_{n}-z_{0}\right)\right\|^{2}+2 \alpha_{n}\left\langle u-z_{0}, x_{n+1}-z_{0}\right\rangle \\
& \leq \beta_{n}\left\|P_{C}(I-\lambda A) x_{n}-z_{0}\right\|^{2}+\gamma_{n}\left\|S_{n} P_{C}(I-\lambda A) u_{n}-z_{0}\right\|^{2}+2 \alpha_{n}\left\langle u-z_{0}, x_{n+1}-z_{0}\right\rangle \\
& \leq \beta_{n}\left\|x_{n}-z_{0}\right\|^{2}+\gamma_{n}\left\|T_{r_{n}} x_{n}-z_{0}\right\|^{2}+2 \alpha_{n}\left\langle u-z_{0}, x_{n+1}-z_{0}\right\rangle \\
& \leq\left(1-\alpha_{n}\right)\left\|x_{n}-z_{0}\right\|^{2}+2 \alpha_{n}\left\langle u-z_{0}, x_{n+1}-z_{0}\right\rangle .
\end{aligned}
$$

From Step 4 and Lemma 2.3, we obtain that $\left\{x_{n}\right\}$ converse strongly to $z_{0}=P_{\mathfrak{F}} u$. By using (3.21), we have $\left\{u_{n}\right\}$ converse strongly to $z_{0}=P_{\mathfrak{F}} u$.

\section{Application}

Using our main theorem (Theorem 3.1), we obtain the following strong convergence theorems involving infinite family of $\kappa$-strict pseudocontractions. as follows.

To prove strong convergence theorem in this section, we need definition and lemma

Definition 4.1. A mapping $T: C \rightarrow C$ is said to be a $\kappa$-strongly pseudocontraction mapping, if there exists $\kappa \in[0,1)$, such that

$$
\|T x-T y\|^{2} \leq\|x-y\|^{2}+\kappa\|(I-T) x-(I-T) y\|^{2}, \quad \forall x, y \in C .
$$

Lemma 4.2 (see [15]). Let $C$ be a nonempty closed convex subset of a real Hilbert space $H$ and $T: C \rightarrow C$ a $\kappa$-strict pseudocontraction. Define $S: C \rightarrow C$ by $S x=\alpha x+(1-\alpha)$ Tx for each $x \in C$. Then, as $\alpha \in[\kappa, 1) S$ is nonexpansive, such that $F(S)=F(T)$.

Theorem 4.3. Let $C$ be a nonempty closed convex subset of a Hilbert space $H$. Let $F$ be bifunctions from $C \times C$ into $\mathbb{R}$ satisfying (A1)-(A4). Let $A: C \rightarrow H$ be a $\alpha$-inverse-strongly monotone mapping. Let $\left\{T_{i}\right\}_{i=0}^{\infty}$ be infinite family of $\kappa_{i}$-pseudocontractions mappings with $\mathfrak{F}=$ $\bigcap_{i=1}^{\infty} F\left(T_{i}\right) \cap \operatorname{EP}(F) \cap \operatorname{VI}(C, A)$. Define a mapping $T_{\mathcal{\kappa}_{i}}$ by $T_{\mathcal{K}_{i}}=\kappa_{i} x+\left(1-\kappa_{i}\right) T_{i} x$, for all $x \in C, i \in \mathbb{N}$, and let $\rho_{j}=\left(\alpha_{1}^{j}, \alpha_{2}^{j}, \alpha_{3}^{j}\right) \in I \times I \times I$, where $I=[0,1], \alpha_{1}^{j}+\alpha_{2}^{j}+\alpha_{3}^{j}=1, \alpha_{1}^{j}+\alpha_{2}^{j} \leq b<1$, and $\alpha_{1}^{j}, \alpha_{2}^{j}, \alpha_{3}^{j} \in(0,1)$ for all $j=1,2, \ldots$. For every $n \in \mathbb{N}$, let $S_{n}$ and $S$ be $S$-mappings generated by $T_{\kappa_{n}}, \ldots, T_{\kappa_{1}}$ and $\rho_{n}, \rho_{n-1}, \ldots, \rho_{1}$ and $T_{\kappa_{n}}, T_{\kappa_{n-1}}, \ldots$, and $\rho_{n}, \rho_{n-1}, \ldots$, respectively. Let $\left\{x_{n}\right\},\left\{u_{n}\right\}$ be 
sequences generated by $x_{1}, u \in C$ and

$$
\begin{gathered}
F\left(u_{n}, y\right)+\frac{1}{r_{n}}\left\langle y-u_{n}, u_{n}-x_{n}\right\rangle \geq 0, \quad \forall y \in C, \\
x_{n+1}=\alpha_{n} u+\beta_{n} P_{C}(I-\lambda A) x_{n}+\gamma_{n} S_{n} P_{C}(I-\lambda A) u_{n}, \quad \forall n \geq 1,
\end{gathered}
$$

where $\left\{\alpha_{n}\right\},\left\{\beta_{n}\right\},\left\{\gamma_{n}\right\} \in(0,1)$, such that $\alpha_{n}+\beta_{n}+\gamma_{n}=1, \beta_{n} \in[c, d] \subset(0,1) r_{n} \in[a, b] \subset$ $(0,2 \alpha), \lambda \subset(0,2 \alpha)$. Assume that

(i) $\lim _{n \rightarrow \infty} \alpha_{n}=0$ and $\sum_{n=0}^{\infty} \alpha_{n}=\infty$,

(ii) $\sum_{n=1}^{\infty} \alpha_{1}^{n}<\infty$,

(iii) $\sum_{n=1}^{\infty}\left|r_{n+1}-r_{n}\right|, \sum_{n=1}^{\infty}\left|\gamma_{n+1}-\gamma_{n}\right|, \sum_{n=1}^{\infty}\left|\alpha_{n+1}-\alpha_{n}\right|, \sum_{n=1}^{\infty}\left|\beta_{n+1}-\beta_{n}\right|<\infty$,

then the sequence $\left\{x_{n}\right\},\left\{u_{n}\right\}$ converges strongly to $z=P_{\mathfrak{F}} u$.

Proof. For every $i \in \mathbb{N}$, by Lemma 4.2, we have that $T_{\kappa_{i}}$ is nonexpansive mappings. From Theorem 3.1, we could have the desired conclusion.

\section{References}

[1] K. Goebel and W. A. Kirk, Topics in Metric Fixed Point Theory, vol. 28 of Cambridge Studies in Advanced Mathematics, Cambridge University Press, Cambridge, UK, 1990.

[2] E. Blum and W. Oettli, "From optimization and variational inequalities to equilibrium problems," The Mathematics Student, vol. 63, no. 1-4, pp. 123-145, 1994.

[3] P. L. Combettes and S. A. Hirstoaga, "Equilibrium programming in Hilbert spaces," Journal of Nonlinear and Convex Analysis, vol. 6, no. 1, pp. 117-136, 2005.

[4] A. Moudafi and M. Théra, "Proximal and dynamical approaches to equilibrium problems," in Ill-Posed Variational Problems and Regularization Techniques (Trier, 1998), vol. 477 of Lecture Notes in Economics and Mathematical Systems, pp. 187-201, Springer, Berlin, Germany, 1999.

[5] H. Iiduka and W. Takahashi, "Weak convergence theorems by Cesáro means for nonexpansive mappings and inverse-strongly-monotone mappings," Journal of Nonlinear and Convex Analysis, vol. 7, no. 1, pp. 105-113, 2006.

[6] H. H. Bauschke and J. M. Borwein, "On projection algorithms for solving convex feasibility problems," SIAM Review, vol. 38, no. 3, pp. 367-426, 1996.

[7] P. L. Combettes, "The foundations of set theoretic estimation," Proceedings of the IEEE, vol. 81, pp. 182-208, 1993.

[8] A. Kangtunyakarn and S. Suantai, "A new mapping for finding common solutions of equilibrium problems and fixed point problems of finite family of nonexpansive mappings," Nonlinear Analysis: Theory, Methods E Applications, vol. 71, no. 10, pp. 4448-4460, 2009.

[9] V. Colao, G. Marino, and H.-K. Xu, "An iterative method for finding common solutions of equilibrium and fixed point problems," Journal of Mathematical Analysis and Applications, vol. 344, no. 1, pp. 340$352,2008$.

[10] S. Takahashi and W. Takahashi, "Viscosity approximation methods for equilibrium problems and fixed point problems in Hilbert spaces," Journal of Mathematical Analysis and Applications, vol. 331, no. 1, pp. 506-515, 2007.

[11] W. Takahashi, Nonlinear Functional Analysis, Fixed Point Theory and Its Applications, Yokohama Publishers, Yokohama, 2000.

[12] F. E. Browder, "Nonlinear operators and nonlinear equations of evolution in Banach spaces," in Nonlinear Functional Analysis (Proc. Sympos. Pure Math., Vol. 18, Part 2, Chicago, Ill., 1968), pp. 1-308, American Mathematical Society, Providence, RI, USA, 1976.

[13] H. K. Xu, "An iterative approach to quadratic optimization," Journal of Optimization Theory and Applications, vol. 116, no. 3, pp. 659-678, 2003. 
[14] W. Takahashi, Introduction to Nonlinear and Convex Analysis, Yokohama Publishers, Yokohama, Japan, 2009.

[15] H. Zhou, "Convergence theorems of fixed points for $\kappa$-strict pseudo-contractions in Hilbert spaces," Nonlinear Analysis: Theory, Methods \& Applications, vol. 69, no. 2, pp. 456-462, 2008. 


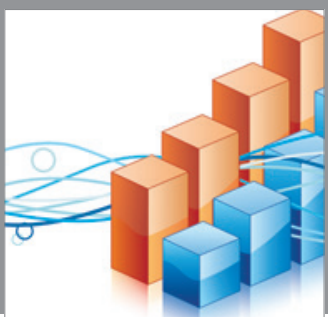

Advances in

Operations Research

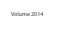

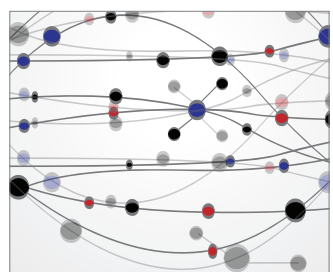

\section{The Scientific} World Journal
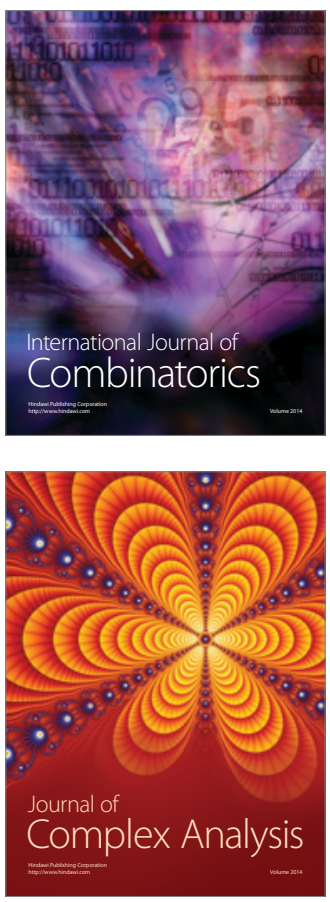

International Journal of

Mathematics and

Mathematical

Sciences
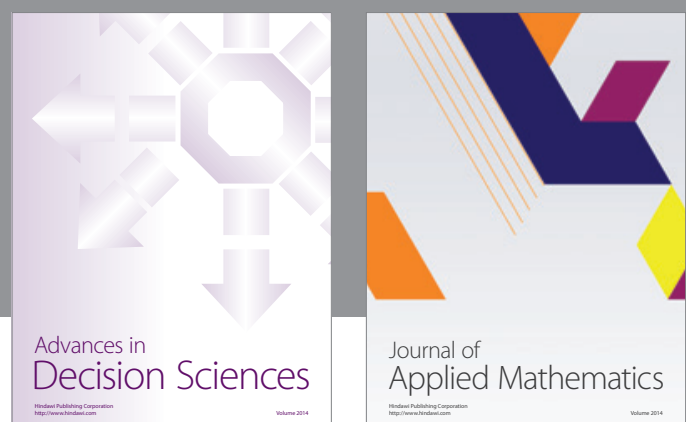

Journal of

Applied Mathematics
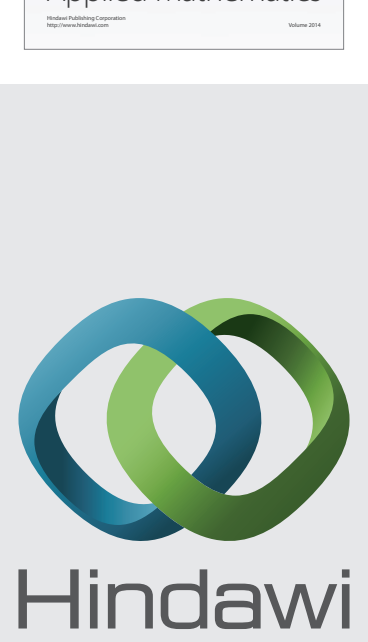

Submit your manuscripts at http://www.hindawi.com
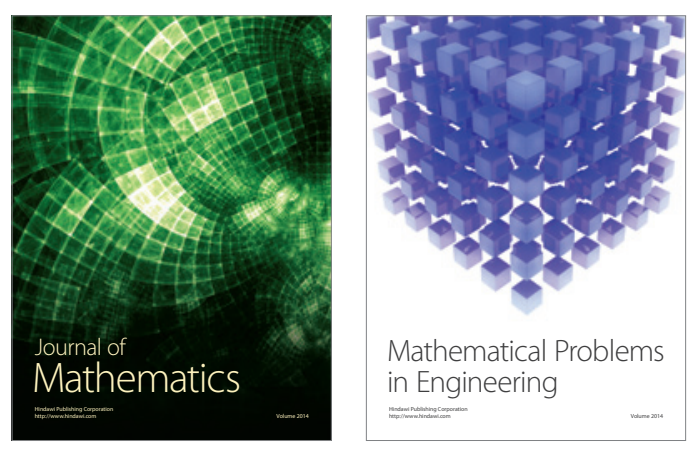

Mathematical Problems in Engineering
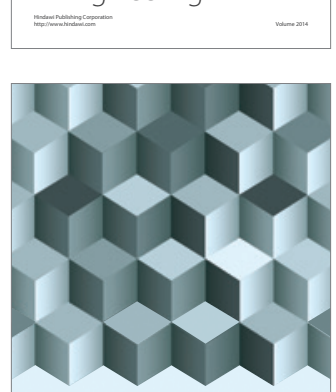

Journal of

Function Spaces
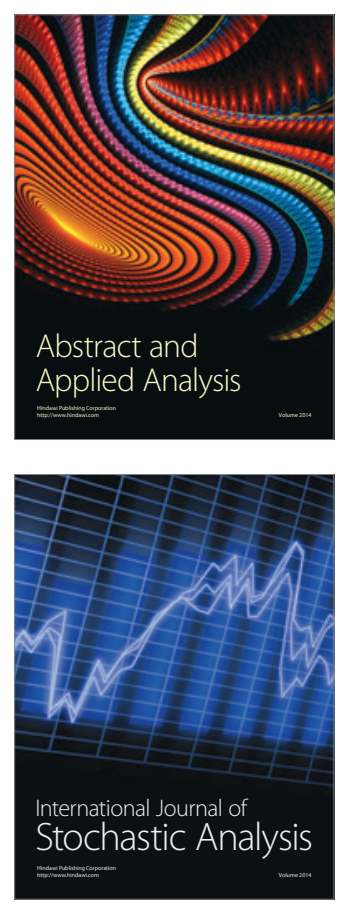

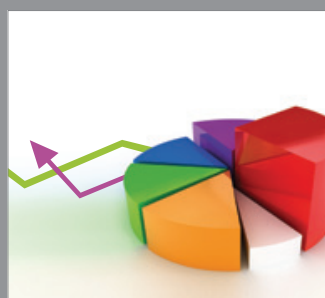

ournal of

Probability and Statistics

Promensencen
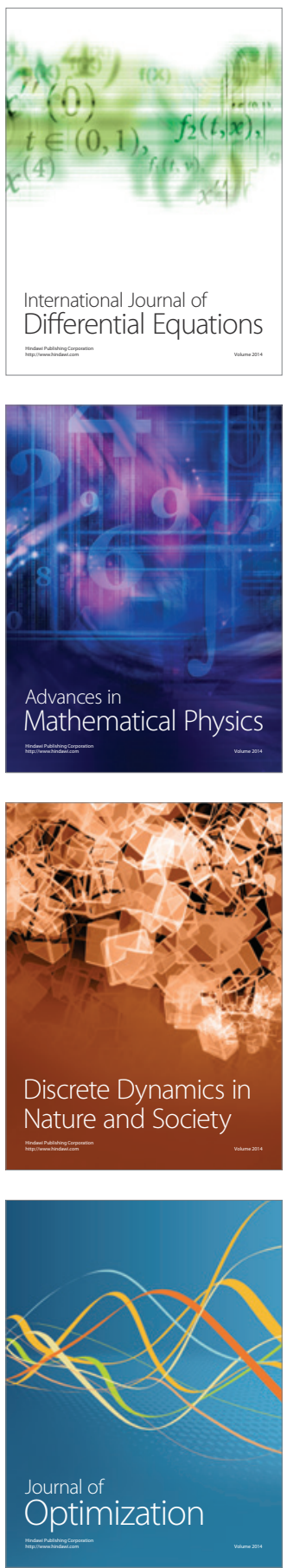